\title{
Consensos de professores especialistas de subáreas de conhecimento de Enfermagem: objetivos, conteúdos e métodos
}

\author{
Consensus of expert professors of subfields of Nursing knowledge: objectives, contents and methods \\ Consenso de profesores especialistas en subáreas de conocimiento de Enfermería sobre objetivos, \\ contenidos y métodos
}

\begin{abstract}
Jouhanna do Carmo Menegaz ${ }^{1,2}$ (D) Stelacelly Coelho Toscano de Brito Silveira ${ }^{1}$ (1)

Vânia Marli Schubert Backes ${ }^{3}$ (1) Carine Vendruscolo ${ }^{2}$ (D)
\end{abstract}

1. Universidade Federal do Pará, Programa de Pós-Graduação em Enfermagem. Belém, PA. Brasil.

2. Universidade do Estado de Santa Catarina, Departamento de Enfermagem. Chapecó, SC, Brasil.

3. Universidade Estadual de Maringá, Programa de Pós-Graduação em Enfermagem. Maringá, PR, Brasil.
Autor correspondente:

Jouhanna do Carmo Menegaz.

E-mail: jouhanna.menegaz@udesc.br

Recebido en 10/08/2021.

Aprovado en 09/01/2022.

\section{Resumo}

Objetivo: descrever as relações entre objetivos, conteúdos e métodos de ensino em subáreas de conhecimento de Enfermagem a partir do consenso de professores especialistas. Método: estudo Delphi em quatro rodadas. Participaram 112 professores de cursos de graduação de Instituições de Ensino Superior públicas do Brasil divididos em sete painéis por subárea. Os dados foram analisados pela técnica de Análise de Conteúdo à luz dos conceitos de conhecimento dos objetivos e conhecimento pedagógico de conteúdo de Shulman, transformados nas variáveis qualitativas de interesse, objetivos, conteúdos e métodos de ensino em torno das quais foi buscado consenso. Resultados: somente as subáreas Enfermagem em Saúde Coletiva e Enfermagem na Gestão e Gerenciamento acordaram objetivos. Conteúdos acertados foram relacionados às políticas públicas e aos programas do Sistema Único de Saúde. Métodos sugerem a coexistência de tradição e inovação. Conclusões e implicações para a prática: a tomada de consciência das relações entre os objetivos, conteúdos e métodos utilizados pelos professores impacta a promoção do diálogo e a integração entre as subáreas, implicando, na prática, uma formação profissional potencializada ou frágil em qualidade resolutiva de problemas de saúde.

Palavras-chave: Docentes; Educação em Enfermagem; Educação Superior; Enfermagem; Ensino.

\section{Abstract}

Aim: to describe the relationships among objectives, content, and teaching methods in subfields of Nursing knowledge from the consensus of expert professors. Methods: a Delphi study in four rounds. Participants were 112 professors from undergraduate courses at public Higher Education Institutions in Brazil divided into seven panels by subarea. The data were analyzed using the Content Analysis technique in light of Shulman's concepts of knowledge of objectives and pedagogical knowledge of content, transformed into the qualitative variables of interest, objectives, content and teaching methods, around which consensus was sought. Results: only the subareas of Collective Health Nursing and Nursing in Management and Administration agreed on objectives. Agreed contents were related to public policies and programs of the Unified Health System. Methods suggest the coexistence of tradition and innovation. Conclusions and implications for practice: the awareness of the relationships among the objectives, contents, and methods used by the professors impacts the promotion of dialogue and the integration among the subareas, implying, in practice, a potentiated or fragile professional formation in resolute quality of health problems.

Keywords: Faculty; Education in Nursing; Higher Education; Nursing; Teaching.

\section{REsumen}

Objetivo: describir las relaciones entre objetivos, contenidos y métodos de enseñanza en subáreas del conocimiento de Enfermería a partir del consenso de profesores especialistas. Método: estudio Delphi en cuatro rondas. Participaron 112 profesores de cursos de pregrado de instituciones públicas de educación superior de Brasil, divididos en siete paneles por subárea. Los datos fueron analizados mediante la técnica de Análisis de Contenido a la luz de los conceptos de conocimiento de los objetivos y conocimiento pedagógico del contenido de Shulman, transformados en variables cualitativas de interés, objetivos, contenidos y métodos de enseñanza en torno a los cuales se buscó el consenso. Resultados: solo las subáreas Enfermería en salud colectiva y Enfermería en gestión y Gerenciamiento acordaron objetivos. Los contenidos del consenso se relacionaron con las políticas públicas y los programas del Sistema Único de Salud. Los métodos sugieren la coexistencia de la tradición e innovación. Conclusiones e implicaciones para la práctica: la conciencia de la relación entre los objetivos, contenidos y métodos empleados por los docentes incide en la promoción del diálogo y la integración entre las subáreas, implicando en la práctica una formación profesional potencializada o frágil en términos de resolución de problemas de salud.

Palabras clave: Docentes; Educación en Enfermería; Educación Superior; Enfermería; Enseñanza. 


\section{INTRODUÇÃO}

A prática dos docentes é sustentada por um conjunto de fontes e conhecimentos base, ${ }^{1}$ que são articulados entre si em um movimento ativo e reflexivo que Shulman ${ }^{2}$ denominou de Modelo de Ação e Raciocínio Pedagógico (MARP). Assim, quatro fontes de conhecimento (formação acadêmica, estrutura e materiais pedagógicos, literatura científica relacionada ao ensino e sabedoria adquirida com a prática docente) subsidiam sete categorias de conhecimento base: conhecimento de conteúdo; conhecimento pedagógico geral; conhecimento pedagógico de conteúdo; conhecimento do currículo; conhecimento sobre os alunos e suas características; conhecimento do contexto educativo e conhecimento dos objetivos, finalidades, valores educativos e seus fundamentos histórico-filosóficos. ${ }^{2,3}$

O MARP sustenta-se tanto em fontes de conhecimento base, que o professor acessa ao longo da trajetória, ${ }^{4}$ quanto no potencial reflexivo do professor. ${ }^{5}$ Assim, em suas fases de compreensão, transformação, ensino, avaliação, reflexão e novas formas de compreender, é distinto a cada professor e pode ser analisado por meio da manifestação do pensar e observação do agir.

A fase de compreensão é o ponto de partida. Nela, podem estar envolvidas todas as categorias de conhecimento base, com destaque aos conhecimentos dos objetivos, pedagógico geral, e de conteúdo. ${ }^{4} \mathrm{O}$ conhecimento dos objetivos, finalidades, valores educativos e seus fundamentos histórico-filosóficos caracteriza o entendimento dos professores sobre por que e para que formar. É mescla de aspectos gerais, como o perfil profissional desejado, e aspectos estritos, como os objetivos de uma disciplina ou aula ${ }^{2}$ e pode ser observado nos planos de ensino, de aula, de forma explícita, ou ainda no discurso e nas escolhas metodológicas dos professores, de forma tácita. ${ }^{6}$

Já o conhecimento pedagógico geral caracteriza as estratégias abrangentes utilizadas pelos professores que transcendem a matéria ou disciplina, enquanto o conhecimento pedagógico de conteúdo é observado na compreensão e ensino por meio da eleição de métodos entendidos como a melhor forma de ensinar e de se aprender em um tema específico, que, no contexto da formação organizada nas subáreas do conhecimento, envolverá as particularidades do conteúdo em relação aos objetivos. ${ }^{7}$

Ao analisar estes conhecimentos no âmbito da fase de compreensão de professores de Enfermagem, observa-se que, para ensinar, os professores sustentam sua prática em diferentes fontes, utilizando evidências de práticas e crenças sobre as melhores formas de ensinar e aprender. ${ }^{8}$ Há estreita relação entre aquilo que os professores acreditam e sabem e o que executam e dominam. ${ }^{7}$ Além de conhecimentos teóricos, técnicos ou científicos adquiridos em sua trajetória de formação acadêmica ou por meio de estruturas e materiais pedagógicos, conhecimentos e crenças obtidos de outras fontes de conhecimento base para o ensino, ${ }^{8}$ como, por exemplo, a sabedoria adquirida com a prática docente, podem também influenciar a ampliação dos objetivos curriculares.

Na formação de enfermeiros, advoga-se em favor do perfil generalista, humanista, crítico-reflexivo, com profissionais habilitados a transformar a realidade social e a ser agentes de mudanças na reorganização das práticas - perfil este descrito nas Diretrizes Curriculares Nacionais (DCNs), ordenadoras da formação no país. ${ }^{9}$

Para tal, se considerada a premissa de que, na relação entre a educação superior e a produção de conhecimento por meio da investigação, esta exerce impacto mais ou menos direto pelas características do currículo, ${ }^{10}$ pode-se considerar que o trabalho pedagógico de escolas e professores se estrutura também, em maior ou menor medida, no conhecimento de Enfermagem, atualmente organizado nas subáreas: Enfermagem Fundamental (EF); Enfermagem de Doenças Emergentes, Reemergentes e Negligenciadas (EDERN); Enfermagem em Saúde do Adulto e do Idoso (ESAI); Enfermagem em Saúde da Mulher (ESMu); Enfermagem na Saúde da Criança e Adolescente (ESCA); Enfermagem na Gestão e Gerenciamento (EGG); Enfermagem em Saúde Mental (ESMe); Enfermagem em Saúde Coletiva (ESC). ${ }^{11}$

Desde a publicação das DCNs, observa-se, nas bases de dados, a contínua publicação de trabalhos que reportam dificuldades para a reorientação da formação profissional em Enfermagem, a exemplo da integração ensino-serviço ${ }^{12}$ e da interdisciplinaridade e transversalidade dos conteúdos relacionados ao Sistema Único de Saúde (SUS). ${ }^{13}$ É possível que estas e outras questões relacionem-se com o fato de que o ensino de Enfermagem intenciona a formação de profissionais generalistas, formados, via de regra, por especialistas que, para além do currículo formal, têm autonomia para mediar objetivos e conteúdos por meio de métodos de ensino.

Ao assumir que, em última instância, se intenciona promover aprendizado para a formação de enfermeiros generalistas, e que o aprendizado se sustenta na compreensão de professores especialistas, que traduzem conhecimento especializado a partir de um MARP individual para um contexto de ensino compartilhado entre pares, o foco deste artigo foi a relação entre objetivo, conteúdo e métodos de ensino. Assim, questiona-se: "Quais as relações entre os objetivos, conteúdos e métodos de ensino acordados por professores especialistas em subáreas do conhecimento de Enfermagem?".

Esta pesquisa tem, portanto, o objetivo de identificar as relações entre objetivos, conteúdos e métodos de ensino em subáreas de conhecimento de Enfermagem a partir do consenso de professores especialistas.

\section{MÉTODO}

Estudo Delphi ${ }^{14}$ eleito em função da possibilidade de considerar professores especialistas de diversas regiões do Brasil, sem a necessidade de reuni-los em um ambiente físico, desenvolvendo o processo por meio de um ambiente virtual. O desenvolvimento do método é sistematicamente comunicacional. Trata-se de uma atividade interativa, controlada pelo pesquisador, com o objetivo de obter o mais confiável consenso de opiniões de um grupo de especialistas, mediante uma série de questionários intensivos, intercalados por feedbacks por meio de respostas curtas relativas às opiniões expostas. ${ }^{15}$

A identificação dos potenciais participantes ocorreu de março a dezembro de 2017 e foi dividida em três etapas: (1) levantamento, via e-MEC, das Instituições de Ensino Superior (IES) públicas 
que ofertavam cursos de graduação em Enfermagem no Brasil; (2) identificação dos professores por subárea do conhecimento a partir dos sítios institucionais dos cursos ou por contato de e-mail; (3) checagem do atendimento aos critérios estabelecidos no estudo e descritos a seguir por meio do Currículo Lattes.

Identificaram-se 79 IES que ofertavam 98 cursos: 19 no Sul; 24 no Sudeste; 17 no Centro-Oeste; 26 no Nordeste e 12 no Norte. Para a consideração do professor como especialista, foram critérios de inclusão: ministrar disciplina em cursos de Enfermagem; possuir graduação em Enfermagem, pós-graduação Lato ou Sricto sensu em subárea do conhecimento de Enfermagem; ter experiência assistencial, gerencial ou técnica como enfermeiro de, pelo menos, dois anos e atuação como docente de Ensino Superior em Enfermagem de, no mínimo, cinco anos. Estabeleceram-se como critérios de exclusão: ser professor temporário e/ou ministrar disciplinas do ciclo básico, como anatomia, biologia etc.

Identificou-se um total geral de 2716 professores assim distribuídos por subárea: 171 em ESMe; 225 em EGG; 254 em EF; 304 em ESCA; 335 em ESMu; 563 em ESC e 731 em ESAI. $\mathrm{Na}$ vigência da identificação dos participantes, a divulgação científica das subáreas do conhecimento eram distintas. Foi utilizada, no estudo, a proposta de Oliveira, ${ }^{16}$ que não inclui EDERN. Foram identificados 133 professores que ministravam disciplinas em mais de uma subárea, alocados como multi e, posteriormente, excluídos. Os professores foram ranqueados para identificar os que atendiam ao maior número de critérios de inclusão, mantendo a proporção regional, na intenção de garantir que todas as regiões do país estivessem contempladas.

Dez por cento dos professores de cada subárea compuseram a amostra por painéis. Subáreas com até 200 professores constituíram painel com 20 professores especialistas; até 300 , com 30 e, assim, sucessivamente, sendo o número máximo por painel de 50 professores. Em cada subárea, os professores foram sendo convidados por e-mail disparado via SurveyMonkey ${ }^{\circledR}$, plataforma online de pesquisa, de acordo com o ranqueamento estabelecido e, à medida que assentiam, recebiam o termo de consentimento e o questionário da primeira rodada. Quando declinavam da participação, eram imediatamente substituídos pelo próximo professor ranqueado na subárea.

Mediante os convites, 260 professores aceitaram participar do estudo. Todavia, 112 responderam ao questionário da primeira rodada, tornando-se efetivamente participantes, representando um retorno de $56 \%$. A coleta de dados ocorreu de janeiro de 2018 a abril de 2019, tendo em vista a abrangência nacional do estudo, e foi desenvolvida em quatro rodadas independentes por subárea, sendo a primeira qualitativa e as seguintes quantitativas, baseadas na rodada imediatamente anterior.

O questionário da primeira rodada continha quatro questões abertas, baseadas nos conceitos de Shulman. ${ }^{2}$ O conteúdo qualitativo da rodada 1 foi analisado por meio da técnica de Análise de Conteúdo ${ }^{17}$ de forma dedutiva, à luz dos construtos de Shulman. ${ }^{2}$ As unidades de registro foram transformadas em variáveis apresentadas nos questionários das rodadas 2,3 e 4 como questões de múltipla escolha ou do tipo caixas de seleção com a intenção de obter consenso acima de $70 \%,{ }^{18}$ calculado por média aritmética. A cada rodada, eram compilados os dados e apresentados aos participantes previamente ao momento de resposta das rodadas seguintes. A obtenção de consensos ocorreu nas subáreas em rodadas diferentes.

Este manuscrito é recorte do macroprojeto de pesquisa "Ação e raciocínio pedagógico de professores de universidades públicas em subáreas do conhecimento de Enfermagem". Apresentam-se os resultados das três variáveis qualitativas nominais destacadas no Quadro 1, obtidas nas rodadas quantitativas. O estudo foi aprovado por Comitê de Ética em Pesquisa sob o Parecer $\mathrm{n}^{\circ} 2.106 .483 / 2017$.

Quadro 1. Rodada, referencial teórico e variáveis de interesse.

\begin{tabular}{|c|c|c|}
\hline Pergunta Rodada 1 & Intenção & Rodadas 2, 3, 4 \\
\hline $\begin{array}{l}\text { Em que você se baseia para } \\
\text { ensinar? }\end{array}$ & $\begin{array}{l}\text { Identificar as fontes de conhecimento } \\
\text { base. }^{2}\end{array}$ & $\begin{array}{l}\text { Busca de consenso sobre as fontes de } \\
\text { conhecimento base } \text { bencionadas pelos }^{2} \\
\text { professores. }\end{array}$ \\
\hline $\begin{array}{l}\text { Na sua subárea, o que você } \\
\text { precisa saber para ensinar? }\end{array}$ & $\begin{array}{l}\text { Identificar o conhecimento de conteúdo² } \\
\text { relacionado à subárea considerado } \\
\text { importante ao ensino de enfermeiros } \\
\text { generalistas. }\end{array}$ & $\begin{array}{l}\text { Variável conteúdo: conhecimentos, } \\
\text { habilidades e atitudes que os professores } \\
\text { da subárea devem ensinar para que os } \\
\text { estudantes atinjam o objetivo. }\end{array}$ \\
\hline $\begin{array}{l}\text { Como você compreende o ensino } \\
\text { na sua área? }\end{array}$ & $\begin{array}{l}\text { Identificar o conhecimento dos objetivos, }{ }^{2} \\
\text { no âmbito da fase de compreensão, }{ }^{2} \text { para } \\
\text { a busca de consenso sobre os objetivos } \\
\text { de ensino na subárea, tendo em vista a } \\
\text { formação de enfermeiros generalistas. }\end{array}$ & $\begin{array}{l}\text { Variável objetivo do ensino na subárea: } \\
\text { intenção explícita do que um estudante } \\
\text { deve desenvolver no escopo da subárea } \\
\text { do conhecimento por meio de uma ou } \\
\text { mais disciplinas. }\end{array}$ \\
\hline $\begin{array}{l}\text { Como você conduz sua disciplina? } \\
\text { Desde o momento em que está se } \\
\text { preparando, estruturando o plano } \\
\text { de ensino, escolhendo métodos de } \\
\text { ensino e avaliação. }\end{array}$ & $\begin{array}{l}\text { Identificar conhecimento pedagógico } \\
\text { geral }\left.\right|^{2} \text { e pedagógico de conteúdo nas fases } \\
\text { de transformação }{ }^{2} \text { e avaliação. }\end{array}$ & $\begin{array}{l}\text { Variável métodos de ensino: descrição } \\
\text { ou sistematização de uma determinada } \\
\text { forma de ensinar com vistas a atingir os } \\
\text { objetivos. }\end{array}$ \\
\hline
\end{tabular}

Fonte: as autoras. 


\section{RESULTADOS}

\section{Breve caracterização das fontes de conhecimento dos participantes}

Quanto à formação acadêmica, $100 \%(n=112)$ dos professores possuíam mestrado, $84 \%$ tinham doutorado $(n=94)$ e $30 \%(n=34)$ dos doutores perpetuaram a qualificação do conhecimento em estágio pós-doutoral; $78 \%$ ( $n=87)$ possuíam experiência profissional como enfermeiro, sendo a subárea de ESCA a única em que todos preenchiam este critério. Quanto à experiência docente, $4,5 \%(n=5)$ possuíam até cinco anos de atuação; $44 \%(n=49)$, até 15 anos; $40 \%(n=45)$, até 30 anos e $11,5 \%(n=13)$, até 45 anos.

\section{Objetivos, conteúdos e métodos de ensino}

Houve consenso, sobretudo, em conteúdos e métodos. Alguns conteúdos foram consenso em mais de um painel, como a sistematização da assistência, o Processo de Enfermagem e a consulta de Enfermagem. O mesmo com os métodos aula expositiva dialogada, simulação e práticas nos serviços de saúde.
Sobre os objetivos, considerando os sete painéis, somente EGG e ESC obtiveram consenso. Ainda que o método eleito para este estudo intencione a obtenção de consensos, optase por apresentar os objetivos delineados nos painéis das demais subáreas pelo entendimento de que dizem respeito à compreensão de parte dos professores no âmbito do MARP e que sua socialização possibilita tecer relações iniciais com os conteúdos e métodos.

As subáreas de ESMe e EGG compartilham o sentido de formação para a atuação em equipe, centrada no usuário e em seus direitos, esta última característica também sinalizada em ESC. A ESMe e a ESC sinalizam as políticas públicas de saúde e as redes de atenção. Há também sinalizações de uma formação em Enfermagem que articula uma ideia de formação integral do enfermeiro (assistência, educação, gestão e investigação) em EGG e ESAl, quando apontam que deve esta articular os processos de trabalho gerencial e assistencial, e a ESAl destaca ainda, no objetivo, a educação em saúde.

No Quadro 2, apresentam-se, por subárea, os consensos obtidos nas variáveis objetivo, conteúdos e métodos de ensino, organizados em sequência, do maior para o menor percentual de consenso.

Quadro 2. Resultados por subárea, por variável.

\begin{tabular}{|c|c|c|c|}
\hline Subárea & Objetivos & Conteúdos & Métodos \\
\hline \multirow{6}{*}{$\mathrm{EF}$} & Um, sem consenso. & 1. Administração de medicamentos (100\%). & 1. Simulação (100\%). \\
\hline & & 2.Técnicas básicas de biossegurança (86\%). & 2. Estudo de casos (86\%). \\
\hline & $\begin{array}{l}\text { Fornecer as bases biológicas, } \\
\text { sociais, assistenciais e ético- } \\
\text { profissionais da Enfermagem (50\%). }\end{array}$ & $\begin{array}{l}\text { 3.Teoria das necessidades humanas básicas } \\
(86 \%) \text {. }\end{array}$ & $\begin{array}{l}\text { 3. Problem Based } \\
\text { Learning ( } 71 \%) \text {. }\end{array}$ \\
\hline & & $\begin{array}{l}\text { 4. Assistência de Enfermagem nas } \\
\text { necessidades de oxigenação, termorregulação } \\
\text { e circulação sanguínea ( } 86 \%) \text {. }\end{array}$ & \\
\hline & & $\begin{array}{l}\text { 5. Assistência de Enfermagem nas } \\
\text { necessidades de segurança ( } 86 \%) \text {. }\end{array}$ & \\
\hline & & $\begin{array}{l}\text { 6. Sistematização da Assistência de } \\
\text { Enfermagem (71\%). }\end{array}$ & \\
\hline \multirow{6}{*}{ ESCA } & Um, sem consenso. & $\begin{array}{l}\text { 1. Crescimento e desenvolvimento infantil } \\
(100 \%) \text {. }\end{array}$ & \multirow{6}{*}{ 1. Simulação (70\%). } \\
\hline & & $\begin{array}{l}\text { 2. Cuidado integral e intervenções de Enfermagem } \\
\text { ao recém-nascido, criança e adolescente na } \\
\text { atenção básica e hospitalar (93\%). }\end{array}$ & \\
\hline & $\begin{array}{l}\text { Introduzir o estudante, futuro } \\
\text { profissional, na prática de cuidado à } \\
\text { criança, adolescente e família. }\end{array}$ & $\begin{array}{l}\text { 3. Cuidado e prevenção a fenômenos graves. } \\
\text { Ex.: violência na adolescência, gravidez } \\
\text { precoce, uso e abuso de drogas, bullying } \\
(93 \%) \text {. }\end{array}$ & \\
\hline & & $\begin{array}{l}\text { 4. Doenças e agravos prevalentes na infância e } \\
\text { adolescência (93\%). }\end{array}$ & \\
\hline & & $\begin{array}{l}\text { 5. Necessidades da criança hospitalizada } \\
\text { e suas repercussões para as crianças/ } \\
\text { adolescentes e famílias ( } 93 \%) \text {. }\end{array}$ & \\
\hline & & $\begin{array}{l}\text { 6. Ética e bioética aplicadas ao cuidado de } \\
\text { Enfermagem à criança e do adolescente ( } 80 \%) \text {. }\end{array}$ & \\
\hline
\end{tabular}

Fonte: as autoras. 
Quadro 2. Continuação...

\begin{tabular}{|c|c|c|c|}
\hline Subárea & Objetivos & Conteúdos & Métodos \\
\hline & Quatro, sem consenso. & $\begin{array}{l}\text { 1. Atuação do enfermeiro na atenção à saúde } \\
\text { e doença mental no contexto familiar e } \\
\text { comunitário (100\%). }\end{array}$ & $\begin{array}{l}\text { 1. Discussão em grupos } \\
\text { (100\%). }\end{array}$ \\
\hline & & 2. Projeto terapêutico singular (100\%). & 2. Estudo de casos (91\%). \\
\hline & $\begin{array}{l}\text { 1. Instrumentalizar os estudantes } \\
\text { de Enfermagem para atuar } \\
\text { junto à equipe de atenção } \\
\text { psicossocial, embasados nas } \\
\text { políticas e na atenção aos } \\
\text { quadros psicopatológicos, com } \\
\text { ênfase na compreensão da Rede } \\
\text { de Atenção Psicossocial (RAPS) } \\
\text { (30\%). }\end{array}$ & $\begin{array}{l}\text { 3. História da Psiquiatria e saúde mental } \\
(100 \%) \text {. }\end{array}$ & $\begin{array}{l}\text { 3. Visita a serviços de } \\
\text { saúde ( } 91 \%) \text {. }\end{array}$ \\
\hline & & 4. Rede de atenção psicossocial (91\%). & 4. Aula expositiva (82\%). \\
\hline \multirow[t]{7}{*}{ ESMe } & $\begin{array}{l}\text { 2. Sensibilizar e instrumentar os } \\
\text { estudantes sobre a necessidade } \\
\text { da reinserção social dos } \\
\text { indivíduos, por meio de uma } \\
\text { abordagem centrada na pessoa } \\
\text { e não na doença, para que } \\
\text { possam atuar enaltecendo e } \\
\text { fortalecendo as habilidades } \\
\text { preservadas dos pacientes, } \\
\text { diminuindo as perdas, } \\
\text { estimulando a ressignificação } \\
\text { de vivências e fortalecendo } \\
\text { laços }(20 \%) \text {. }\end{array}$ & 5. Políticas públicas de saúde mental (91\%). & $\begin{array}{l}\text { 5. Exibição de filmes e } \\
\text { vídeos ( } 73 \%) \text {. }\end{array}$ \\
\hline & & $\begin{array}{l}\text { 6. Transtornos mentais. Ex.: esquizofrenia, } \\
\text { transtorno bipolar, etc.) (91\%). }\end{array}$ & \\
\hline & $\begin{array}{l}\text { 3. Apresentar o panorama } \\
\text { histórico da Psiquiatria, a Reforma } \\
\text { Psiquiátrica, os modelos de } \\
\text { atenção em saúde, o cuidado } \\
\text { em rede, diferenciando a } \\
\text { doença mental da saúde mental } \\
\text { e enfatizando a atuação do } \\
\text { enfermeiro e da equipe de saúde } \\
\text { na área ( } 20 \% \text { ). }\end{array}$ & 7. Técnicas de comunicação terapêutica (91\%). & \\
\hline & & $\begin{array}{l}\text { 8. Consulta de Enfermagem em Psiquiatria } \\
(82 \%) \text {. }\end{array}$ & \\
\hline & $\begin{array}{l}\text { 4. Ensinar sobre Enfermagem, } \\
\text { Psiquiatria e Saúde Mental (10\%). }\end{array}$ & $\begin{array}{l}\text { 9. Assistência de Enfermagem em quadros } \\
\text { psicopatológicos (82\%). }\end{array}$ & \\
\hline & & 10. Exame psíquico (73\%). & \\
\hline & & 11. Psicofármacos (73\%). & \\
\hline
\end{tabular}

Fonte: as autoras. 
Quadro 2. Continuação...

\begin{tabular}{|c|c|c|c|}
\hline Subárea & Objetivos & Conteúdos & Métodos \\
\hline \multirow{12}{*}{ ESMu } & Três, sem consenso. & $\begin{array}{l}\text { 1. Patologias prevalentes em saúde da mulher } \\
(100 \%) \text {. }\end{array}$ & $\begin{array}{l}\text { 1. Estudo de casos } \\
(100 \%) \text {. }\end{array}$ \\
\hline & & 2. Fatores de risco à saúde da mulher (100\%). & 2. Aula expositiva (83\%). \\
\hline & $\begin{array}{l}\text { Intervenção assistencial à saúde } \\
\text { da mulher com o intuito de } \\
\text { identificação dos problemas, } \\
\text { promoção da saúde e prevenção de } \\
\text { agravos (50\%). }\end{array}$ & 3. Políticas de saúde da mulher (100\%). & 3. Simulação (83\%). \\
\hline & & 4. SISCOLO/SISMAMA (100\%). & 4. Problematização (83\%). \\
\hline & $\begin{array}{l}\text { Desenvolver competências e } \\
\text { habilidades para a Atenção Integral } \\
\text { à Saúde da Mulher no âmbito do } \\
\text { SUS (33\%). }\end{array}$ & $\begin{array}{l}\text { 5. Prevenção e detecção precoce do câncer } \\
\text { cervicouterino e de mama (100\%). }\end{array}$ & \\
\hline & & $\begin{array}{l}\text { 6. Prevenção e detecção precoce das infecções } \\
\text { sexualmente transmissíveis (100\%). }\end{array}$ & \\
\hline & $\begin{array}{l}\text { Promover assistência à saúde da } \\
\text { mulher em seu ciclo vital (17\%). }\end{array}$ & 7. Sexualidade e reprodução (100\%). & \\
\hline & & 8. Planejamento reprodutivo (100\%). & \\
\hline & & 9. Violência Obstétrica (100\%). & \\
\hline & & $\begin{array}{l}\text { 10. Assistência ao aleitamento materno } \\
(100 \%) \text {. }\end{array}$ & \\
\hline & & 11. Aborto (100\%). & \\
\hline & & $\begin{array}{l}\text { 12. Cuidado à mulher durante o trabalho de } \\
\text { parto, parto e puerpério ( } 86 \%) \text {. }\end{array}$ & \\
\hline \multirow[b]{3}{*}{ ESAI } & Um, sem consenso. & 1. Manejo da dor em adultos e idosos (100\%). & \multirow[b]{3}{*}{$\begin{array}{l}\text { 1. Práticas nos serviços } \\
(86 \%) \text {. }\end{array}$} \\
\hline & & $\begin{array}{l}\text { 2. Assistência de Enfermagem a pacientes } \\
\text { com doenças oncohematológicas, do sistema } \\
\text { cardiovascular, respiratório, digestório e } \\
\text { urinário }(86 \%) \text {. }\end{array}$ & \\
\hline & $\begin{array}{l}\text { Ensinar o processo de cuidar em } \\
\text { Enfermagem ao adulto e ao idoso } \\
\text { no seguimento ambulatorial e } \\
\text { hospitalar com afecções agudas } \\
\text { e crônicas. Assistência à família e } \\
\text { cuidadores e o desenvolvimento de } \\
\text { práticas educativas ( } 67 \%) \text {. }\end{array}$ & 3. Hipertensão e Diabetes (86\%). & \\
\hline \multirow{15}{*}{ EGG } & \multirow{15}{*}{$\begin{array}{l}\text { Gerenciar o cuidado global e } \\
\text { de Enfermagem, articulando a } \\
\text { equipe multiprofissional com } \\
\text { a equipe de Enfermagem no } \\
\text { processo de cuidado ao usuário. } \\
\text { Há envolvimento do enfermeiro } \\
\text { na resolução das necessidades } \\
\text { gerais, não somente as de sua } \\
\text { exclusiva competência. Quando } \\
\text { de sua competência, utiliza a } \\
\text { articulação dos processos de } \\
\text { trabalho assistencial e gerencial } \\
\text { em favor da 'integralidade do } \\
\text { pensar e do fazer'. Quando } \\
\text { transcende sua competência, } \\
\text { envolve e corresponsabiliza os } \\
\text { demais profissionais no processo } \\
\text { de cuidado, atuando como um } \\
\text { 'advogado' da pessoa/usuário. }\end{array}$} & 1. Modelos de gestão (100\%). & \multirow{15}{*}{$\begin{array}{l}\text { 1. Metodologias ativas } \\
(91 \%) \text {. }\end{array}$} \\
\hline & & 2. Sistemas de informação (100\%). & \\
\hline & & 3. Teorias da administração (100\%). & \\
\hline & & $\begin{array}{l}\text { 4. Tomada de decisão/processo decisório } \\
(100 \%) \text {. }\end{array}$ & \\
\hline & & 5. Gestão de pessoas (100\%). & \\
\hline & & 6. Trabalho em equipe (100\%). & \\
\hline & & 7. Educação Permanente (100\%). & \\
\hline & & $\begin{array}{l}\text { 8. Planejamento e organização dos serviços } \\
(100 \%) \text {. }\end{array}$ & \\
\hline & & 9. Processo de trabalho (100\%). & \\
\hline & & 10. Dimensionamento de Enfermagem (92\%). & \\
\hline & & 11. Liderança (92\%). & \\
\hline & & 12. Empreendedorismo (92\%). & \\
\hline & & 13. Relacionamento Interpessoal (92\%). & \\
\hline & & 14. Supervisão (83\%). & \\
\hline & & 15. Comunicação (83\%). & \\
\hline
\end{tabular}


Quadro 2. Continuação...

\begin{tabular}{|c|c|c|c|}
\hline Subárea & Objetivos & Conteúdos & Métodos \\
\hline \multirow{17}{*}{ ESC } & \multirow{17}{*}{$\begin{array}{l}\text { Formar enfermeiro com } \\
\text { conhecimento do arcabouço } \\
\text { teórico e prático do campo } \\
\text { da saúde coletiva aplicada à } \\
\text { Enfermagem, com a compreensão } \\
\text { sobre o sistema de saúde e seus } \\
\text { determinantes sociais, com vistas a } \\
\text { garantir autonomia e discernimento } \\
\text { para assegurar a integralidade } \\
\text { do atendimento prestado aos } \\
\text { indivíduos, famílias e comunidades, } \\
\text { com qualidade e humanização para } \\
\text { intervir no processo saúde/doença. }\end{array}$} & 1.Epidemiologia (100\%). & $\begin{array}{l}\text { 1.Visitas a serviços de } \\
\text { saúde }(100 \%) \text {. }\end{array}$ \\
\hline & & $\begin{array}{l}\text { 2.Planejamento e Org. dos Serviços de Saúde } \\
(100 \%) \text {. }\end{array}$ & $\begin{array}{l}\text { 2. Práticas nos serviços } \\
(100 \%) \text {. }\end{array}$ \\
\hline & & $\begin{array}{l}\text { 3. Sistemas de Saúde, Políticas Públicas de } \\
\text { Saúde vigentes (100\%). }\end{array}$ & \\
\hline & & 4. Determinantes Sociais de Saúde (100\%). & \\
\hline & & 5. Processos de trabalho em saúde (93\%). & \\
\hline & & 6. Processo saúde/doença (93\%). & \\
\hline & & 7. História da saúde coletiva (93\%). & \\
\hline & & 8. Vigilância em saúde (93\%). & \\
\hline & & 9. Atenção Básica (93\%). & \\
\hline & & 10. Necessidades em saúde (85\%). & \\
\hline & & 11. Redes de saúde (85\%). & \\
\hline & & 12. Indicadores de saúde (85\%). & \\
\hline & & 13. Imunização e redes de frio (85\%). & \\
\hline & & 14. Visita domiciliar (85\%). & \\
\hline & & 15. Níveis de prevenção (85\%). & \\
\hline & & 16. Programas do Ministério da Saúde & \\
\hline & & (77\%). & \\
\hline
\end{tabular}

Fonte: as autoras.

\section{DISCUSSÃO}

Há intencionalidade ao ensinar, como delineou Shulman ${ }^{2}$ no MARP. A partir de um dado conteúdo ou disciplina, este processo inicia-se com movimentos de compreensão e transformação realizados pelo professor em relação a objetivos, conteúdos e métodos de ensino.

Isso posto, buscaram-se identificar, a partir da explicitação do raciocínio pedagógico de professores, possíveis relações entre estes elementos não somente restritos ao âmbito de sua disciplina, mas em um exercício reflexivo de, a partir dela, extrapolar para um contexto mais amplo, o do ensino de uma determinada subárea do conhecimento de Enfermagem no âmbito de uma formação generalista. Assim, ao longo das rodadas da pesquisa, a partir da compressão que possuíam sobre a formação de enfermeiros, considerando suas fontes de conhecimento, os professores acordaram objetivos e elegeram conteúdos e métodos de ensino que consideraram pertinentes para atingi-los.

Sobre os objetivos, observou-se baixo consenso nas e entre as subáreas, o que pode se relacionar ao fato de que isto talvez não seja possível pela multiplicidade de competências requeridas do enfermeiro, cada uma delas gerando objetivos específicos, ou pela miscelânea de compreensões dentro da mesma subárea, reforçando a ideia de que o raciocínio pedagógico, além de individualizado, pode ser pouco compartilhado entre pares e negociado no contexto educacional.

Na tentativa de pensar a formação do enfermeiro de forma mais ampla e não restrita a uma ou outra disciplina, a clareza do objetivo da subárea, na fase de compreensão, parece cara à formação em Enfermagem. Um estudo a respeito do ensino da Enfermagem na América Latina e no Caribe ${ }^{19}$ propôs que currículos de graduação em Enfermagem potencializem atividades integradas e articuladas, a fim de alinhar competências universais e oportunizar, aos alunos, práticas de aprendizagem inovadoras e educação transformadora, valorizando a formação de profissionais que atendam às necessidades da população, ${ }^{20}$ o que implica que exista discussão entre professores de uma mesma subárea e também entre as subáreas.

A despeito das dificuldades de consenso, observam-se, na declaração dos objetivos de forma compartilhada entre algumas subáreas, verbos compatíveis com abordagens centradas no conteúdo, que são, geralmente, também centradas no professor, ${ }^{21}$ como fornecer (EF), introduzir (ESCA), instrumentalizar (ESMe) e ensinar (ESMe e ESAI). Abordagens como as destacadas são caracterizadas pelo fato de que a ação em relação ao objeto de aprendizagem (conteúdo, por exemplo) não é do estudante.

Quando analisados os conteúdos em comum entre as subáreas, observa-se uma negociação de compreensão entre o que seria conteúdo específico e conteúdos relacionados ao 
SUS, suas políticas e programas. Isto pode ser resultado do forte movimento indutor de reorientação da formação profissional em saúde para o SUS, que se concentrou, significativamente, nos primeiros momentos, na mudança das estruturas e dos materiais pedagógicos, alterando projetos pedagógicos e ementas, com dificuldade de reverberar em mudanças de práticas. ${ }^{9}$

Já nos métodos, observa-se a predominância de consensos em torno de métodos relacionados à ideia de aprendizagem ativa, característica de abordagens centradas no estudante, como práticas, estudos de caso e simulação, em conjunto ao método caracterizado como tradicional: a aula expositiva. Neste sentido, é possível que a formação em Enfermagem esteja em um período de transição induzido pelo constante discurso promotor da utilização de metodologias ativas, ainda que o ensino em Enfermagem seja visto como predominantemente tradicional. ${ }^{22}$

É também possível que isto seja um reflexo de um movimento de orientação tecnológica da formação docente ${ }^{5} \mathrm{em}$ que, com base na literatura científica, se sugerem melhores práticas ou melhores métodos ${ }^{23}$ de ensinar, criando métodos canônicos. Esse fato pode sugerir que o conhecimento pedagógico de conteúdo seja instrumental, gerando modelos, formas padrão.

Observa-se também que as subáreas que listaram maior número de objetivos (EF, ESMu, ESMe), ainda que não pactuados, entraram em consenso sobre o maior número de métodos, o que pode sugerir maior diversidade didática na prática dos professores, benéfica à aprendizagem dos estudantes, pois contribui para um maior alcance de estudantes, com diferentes estilos de aprendizagem. ${ }^{24,25}$

Assim, se tomado o encadeamento entre objetivos, conteúdos e métodos, deve-se atentar para a presença de um distanciamento entre a intenção e a ação, pois a redação dos objetivos enfoca o professor e o método e estes requerem a participação ativa do estudante. Novamente, os distanciamentos na relação entre objetivos e métodos podem ser observados entre e nas subáreas, no uso de verbos e substantivos. Educar, ensinar, formar, sensibilizar, instrumentalizar e intervir carregam consigo espectros distintos, os quais podem conduzir os professores à eleição de métodos de ensino mais ou menos diretivos para atingir estes objetivos. ${ }^{26}$

Tomar consciência destes aspectos é relevante para a formação em Enfermagem, especialmente, se adicionada também uma consideração sobre a avaliação. A avaliação é apontada como um desafio do trabalho docente pelos potenciais conflitos teóricos do que avaliar, como avaliar e quem avalia. ${ }^{27}$

Na perspectiva da avaliação no MARP, é nesta fase que os professores avaliam o planejado e executado e, em teoria, é uma fase na qual os dados de desempenho dos estudantes são outputs do seu próprio desempenho. Tal perspectiva confirma que é preciso definir, prévia e claramente, os objetivos e o processo de ensino e avaliação de modo a serem perseguidos e, progressivamente, alcançados nas ações de ensino, como, também, constantemente verificados pela proposição de diferentes atividades avaliativas. ${ }^{28}$

\section{CONCLUSÕES E IMPLICAÇÕES PARA A PRÁTICA}

Uma vez que a formação em Enfermagem segue as DCNs de Ensino, considerando os contextos locais de formação dos professores, é possivel analisar as relações entre objetivos, conteúdos e métodos de ensino, e relacionar o 'fazer' entre e nas subáreas de conhecimento da Enfermagem. Destacou-se que há objetivos centrados no conteúdo e métodos de ensino que sugerem tanto a manutenção quanto a inovação por meio de aproximações com foco que caracteriza a abordagem centrada no estudante.

O método Delphi proporcionou que os professores discutissem esta intencionalidade e seus desdobramentos a partir de suas compreensões particulares, apresentando, assim, dificuldades na obtenção de consensos sobre os objetivos do conhecimento. A clareza dos objetivos auxilia os professores a eleger conteúdos e métodos de ensino compatíveis, bem como pode proporcionar diálogo entre os professores de diferentes subáreas. Isso seria profícuo ao desenvolvimento do ensino na subárea e à educação em Enfermagem, mas, também, com a possível presença de conservadorismo, padronização e inovação coexistindo.

A tomada de consciência do encadeamento entre objetivos, conteúdos e métodos pode colaborar com os resultados de aprendizagem de estudantes e servir de subsídio à formação docente, uma vez que a sabedoria adquirida com a prática de professores é uma importante fonte de conhecimento base para o ensino. Haja vista que as implicações para a prática reverberam em uma formação profissional potencializada ou frágil em qualidade resolutiva de problemas de saúde.

Destacam-se como limitações do estudo as perdas na primeira rodada e o fato de que a análise é baseada nas falas dos professores e não na observação de suas práticas e análise dos planos de aula. Discutem-se os consensos entre os professores por painéis, que não representam, necessariamente, o que fazem. Estudos in loco são recomendados.

\section{CONTRIBUIÇÕES DOS AUTORES}

Desenho do estudo. Jouhanna do Carmo Menegaz.

Coleta ou produção dos dados. Jouhanna do Carmo Menegaz. Stelacelly Coelho Toscano de Brito Silveira.

Análise de dados. Jouhanna do Carmo Menegaz. Stelacelly Coelho Toscano de Brito Silveira. Vânia Marli Schubert Backes. Carine Vendruscolo.

Interpretação dos resultados. Jouhanna do Carmo Menegaz. Stelacelly Coelho Toscano de Brito Silveira. Vânia Marli Schubert Backes. Carine Vendruscolo.

Redação e revisão crítica do manuscrito. Jouhanna do Carmo Menegaz. Stelacelly Coelho Toscano de Brito Silveira. Vânia Marli Schubert Backes. Carine Vendruscolo.

Aprovação da versão final do artigo. Jouhanna do Carmo Menegaz. Stelacelly Coelho Toscano de Brito Silveira. Vânia Marli Schubert Backes. Carine Vendruscolo. 
Responsabilidade por todos os aspectos do conteúdo e a integridade do artigo publicado. Jouhanna do Carmo Menegaz. Stelacelly Coelho Toscano de Brito Silveira. Vânia Marli Schubert Backes. Carine Vendruscolo.

\section{EDITOR ASSOCIADO}

Stela Maris de Melo Padoin (1)

\section{EDITOR CIENTÍFICO}

\author{
Ivone Evangelista Cabral (B)
}

\section{REFERÊNCIAS}

1. Gess-Newsome J, Taylor JA, Carlson J, Gardner AL, Wilson CD, Stuhlsatz MAM. Teacher pedagogical content knowledge, practice, and student achievement. Int J Sci Educ. 2019;41(7):944-63. http://dx.doi.org/10. 1080/09500693.2016.1265158.

2. Shulman LS. Conhecimento e ensino: fundamentos para a nova reforma. Cad Cenpec. 2014;4(2):1-22. http://dx.doi.org/10.18676/ cadernoscenpec.v4i2.293.

3. Backes VMS, Menegaz JC, Miranda FAC, Santos LMC, Cunha AP, Patrício SS. Lee Shulman: contributions to research on teacher training in nursing and health. Texto Contexto Enferm. 2017;26(4):e1080017. https://doi.org/10.1590/0104-07072017001080017.

4. Menegaz JC, Marli Schubert Backes V, Medina Moy JLMM. Communities of practice: influences on pedagogical reasoning and action of nursing professors. Invest Educ Enferm.2018;36(2):e02. http://dx.doi.org/10.17533/ udea.iee.v36n2e02.

5. Menegaz JC, Backes VMS, Becerril LC. Formação docente em enfermagem: trajetória e experiência na constituição do saber e do fazer. In: Backes VMS, Menegaz JC, Moya JLM, organizadores. Formação docente na saúde e enfermagem. Porto Alegre: Moriá; 2019. p. 191-206.

6. Canever BP, Prado ML, Gomes DC, Backes VMS, Jesus BH. Selfknowledge of health teachers: a qualitative exploratory study. Nurse Educ Today.2018 Jun;65:54-9. http://dx.doi.org/10.1016/j.nedt.2018.02.035. PMid:29525487.

7. Kind V, Chan $\mathrm{KKH}$. Resolving the amalgam: connecting pedagogical content knowledge, content knowledge and pedagogical knowledge. Int J Sci Educ. 2019 Mar;41(7):964-78. http://dx.doi.org/10.1080/095 00693.2019.1584931.

8. Menegaz JC, Backes VMS, Moya JLM. Ação e raciocínio pedagógico de professoras de enfermagem: expressões em diferentes contextos educacionais. Texto Contexto Enferm. 2018 Aug;27(3):e2660016. http:// dx.doi.org/10.1590/0104-07072018002660016.

9. Resolução CNE/CES no 3, de 7 de novembro de 2001 (BR). Institui Diretrizes Curriculares Nacionais do Curso de Graduação em Enfermagem. Diário Oficial da União [periódico na internet], Brasília (DF), 9 nov. 2001 [citado 19 abr 2018]. Disponível em: http://portal.mec.gov.br/cne/ arquivos/pdf/CES03.pdf

10. Ortiz FXG, Barnett R. Los límites de la competencia: el conocimiento, la educación superior y la sociedad. Investig Bibl [Internet]. 2008; [citado 6 ago 2020 ];22(46):229-35. Disponível em: http://www.scielo.org.mx/ scielo.php?script=sci_arttext\&pid=S0187-358X2008000300011\&lng =es\&nrm=iso

11. Barros ALBL, Nóbrega MML, Santos RS, Cézar-Vaz MR, Pagliuca LMF. Pesquisa em enfermagem e a modificação da árvore do conhecimento no CNPq: contribuição à ciência. Rev Bras Enferm. 2020;73(1):e20170911. PMid:32049221.

12. Reibnitz KS, Kloh D, Corrêa AB, Lima MM. Reorientação da formação do enfermeiro: análise a partir dos seus protagonistas. Rev Gaúcha
Enferm. 2016;37(spe):e68457. http://dx.doi.org/10.1590/1983-1447.2016. esp.68457. PMid:28380155.

13. Winters Jr F, Prado ML. Heidemann ITSB. Formação em enfermagem orientada aos princípios do Sistema Único de Saúde: percepção dos formandos. Esc Anna Nery. 2016;20(2):248-53. https://doi. org/10.5935/1414-8145.20160033.

14. Keeney $\mathrm{S}$, Hasson F, McKenna $\mathrm{H}$. The Delphi technique in nursing and health research. Oxford: Wiley Blackwell; 2011.

15. Munaretto LF, Corrêa HL, Cunha JAC. A study on the characteristics of the Delphi method and focus group as techniques to obtain data in exploratory research. Rev Adm UFSM. 2013;6(1):9-24. http://dx.doi. org/10.5902/198346596243.

16. Oliveira DC, Ramos FRS, Barros ALBL, Nóbrega MML. Classificação das áreas de conhecimento do CNPq e o campo da Enfermagem: possibilidades e limites. Rev Bras Enferm. 2013;66(spe):60-5. http:// dx.doi.org/10.1590/S0034-71672013000700008. PMid:24092311.

17. Bardin L. Análise de conteúdo. São Paulo: Edições 70; 2016.

18. Castro AV, Rezende M. A técnica Delphi e seu uso na pesquisa de enfermagem: revisão bibliográfica. REME Rev Min Enferm [Internet] 2009; [citado 2020 ago 6];13(3):429-34. Disponível em: http://www. reme.org.br/artigo/detalhes/209

19. Cassiani SHB, Wilson LL, Mikael SSE, Peña LM, Grajales RAZ, McCreary LL et al. The situation of nursing education in Latin America and the Caribbean towards universal health. Rev Lat Am Enfermagem. 2017;25(0):e2913. http://dx.doi.org/10.1590/1518-8345.2232.2913. PMid:28513769.

20. Egry EY. A glance at the good practices of nursing in primary care Rev Bras Enferm. 2018;71(3):930-1. http://dx.doi.org/10.1590/00347167.2018710301. PMid:29924171.

21. Serin H. a comparison of teacher-centered and student-centered approaches in educational settings. Int J Social Sci Educ Stud [Internet] 2018; [citado 2020 ago 6];5(1):164-7. Disponível em: https://ijsses.tiu. edu.iq/index.php/volume-5-issue-1-article-14/

22. Ximenes No FRG, Lopes No D, Cunha ICKO, Ribeiro MA, Freire NP, Kalinowski CE et al. Reflexões sobre a formação em Enfermagem no Brasil a partir da regulamentação do Sistema Único de Saúde. Cien Saude Colet. 2020;25(1):37-46. http://dx.doi.org/10.1590/141381232020251.27702019. PMid:31859853.

23. Alvarado C, Cañada F, Garritz A, Mellado V. Canonical pedagogica content knowledge by CoRes for teaching acid-base chemistry at high school. Chem Educ Res Pract. 2015;16(3):603-18. http://dx.doi. org/10.1039/C4RP00125G.

24. Arghode V, Wang J, Lathan A. Exploring instructors' practices in student engagement: a collective case study. J Scholarsh Teach Learn. 2017;17(4):126-49. http://dx.doi.org/10.14434/v17i4.22099.

25. Stirling BV. Results of a study assessing teaching methods of faculty after measuring student learning style preference. Nurse Educ Today. 2017;55:107-11. http://dx.doi.org/10.1016/j.nedt.2017.05.012. PMid:28575707.

26. Tondeur J, van Braak J, Ertmer PA, Ottenbreit-Leftwich A. Understanding the relationship between teachers' pedagogical beliefs and technology use in education: a systematic review of qualitative evidence. Educ Technol Res Dev. 2017;65(3):555-75. http://dx.doi.org/10.1007/s11423016-9481-2.

27. Bernardi MC, Prado ML, Kempfer SS, Ribeiro KRB, de Oliveira SN. The portfolio in the evaluation of the undergraduate student in the health area: a bibliometric study. Cogitare Enferm. 2015;20(1):152-9. http:// dx.doi.org/10.5380/ce.v20i1.38199.

28. Favarão CFM, Salvi RF. Avaliação da aprendizagem: qual o significado em sala de aula? Anais do XI Seminário de Pesquisa em Ciências Humanas; 2016 jul. 27-29; Londrina (RS), Brasil. Londrina (RS): Centro de Letras e Ciências Humanas, Universidade Estadual de Londrina; 2016. p. 1555-65. 